\title{
O CONTROLE SOCIAL NA ATENÇÃo PRIMÁRIA E A ATUAÇÃo DOS CONSELHOS MUNICIPAIS
}

\author{
Karen de Almeida Barroso ${ }^{1}$ \\ George Sobrinho Silva²
}

BARROSO, K. de A.; SILVA, G. S. O controle social na atenção primária e a atuação dos conselhos municipais. Arq. Cienc. Saúde UNIPAR, Umuarama, v. 19, n. 3, p, 213-219, set./dez. 2015.

RESUMO: O controle social é um mecanismo pelo qual se garante a participação dos usuários na organização, funcionalidade e busca pela qualidade dos serviços de saúde no Brasil. O objetivo deste trabalho foi de conhecer a atuação dos conselheiros municipais de saúde no controle social junto à organização da atenção primária à saúde. Trata-se de um estudo transversal descritivo de abordagem quantitativa realizado com 40 conselheiros de saúde de quatro municípios da microrregião sanitária de Diamantina, Minas Gerais. Utilizando-se um questionário semiestruturado na coleta de dados e sua análise se deu por meio da estatística descritiva. Como resultados observou-se que os conselheiros eram preponderantemente do gênero feminino $(72,5 \%)$, com curso superior $(52,5 \%)$ e com vínculos trabalhistas ligados aos serviços públicos de saúde dos respectivos municípios $(35,0 \%)$. Os conselheiros relatam conhecer os serviços de atenção primária, seus problemas, porém há baixa participação em reuniões nas respectivas unidades de saúde onde são usuários. O estudo evidenciou uma fragilidade do exercício do controle social na atenção primária à saúde e a necessidade de maior aproximação dos conselheiros com estes serviços.

PALAVRAS-CHAVE: Participação social; Conselhos de saúde; Atenção primária à saúde.

\section{SOCIAL CONTROL IN PRIMARY HEALTH AND THE ACTIONS OF MUNICIPAL COUNCILS}

\begin{abstract}
Social control is a mechanism used to ensure the participation of users from the public health services in its organization, functionality and in the search for quality services in Brazil. The objective of this study was to know the performance of municipal health counselors in social control in the primary health care. This is a descriptive cross-sectional study with a quantitative approach carried out with 40 health counselors from four municipalities in the health micro-region of Diamantina, Minas Gerais, Brazil. A semistructured questionnaire was used to collect data. The data was analyzed using descriptive statistics. As a result, it could be observed that the counselors were predominantly female $(72.5 \%)$, college graduates $(52.5 \%)$ and having labor bonds with the public health services in their municipalities $(35.0 \%)$. The counselors reported knowing the meaning of primary care, their services and issues, but there is low participation in meetings in their health services where they are users. The study showed a weakness of social control in primary health care and the need for further approximation of directors with these services.
\end{abstract}

KEYWORDS: Social participation; Health councils; Primary health care.

\section{Introdução}

O setor da saúde foi pioneiro na criação de mecanismos que garantissem a participação da sociedade na gestão dos serviços públicos no Brasil, conforme artigo 198, da Constituição de 1988 (BRASIL, 2013). Esta conquista foi proveniente de movimentos populares, decorrentes da efervescência política do período da ditadura militar, que culminaram na criação do Sistema Único de Saúde (SUS) que tem o controle social como um dos seus princípios doutrinários (CORREA, 2005).

O controle social é garantido por meio da realização de Conferências e dos Conselhos de Saúde que devem existir no âmbito dos três níveis de governo: Federal, Estadual e Municipal (SHIMIZU et al., 2013). Os Conselhos Municipais de Saúde, foco deste estudo, são órgãos colegiados de caráter permanente e deliberativo que devem ser compostos por um conjunto de conselheiros que devem representar paritariamente a sociedade, conforme preconiza a Lei Orgânica da Saúde 8.142/90 (BRASIL, 2015). Em 2012, todos os municípios do país já possuíam Conselhos de Saúde que mobilizavam cerca de 72 mil conselheiros se reunindo pelo menos uma vez por mês para deliberar sobre as suas respectivas demandas (CRISTO, 2012).

Esses conselheiros têm a responsabilidade de atua- rem de forma comprometida com sua comunidade e os seus respectivos serviços de saúde. Sua atuação deve ser baseada nos direitos de cidadania de toda a população, ajudando a planejar as políticas de saúde, fiscalizando como o governo cuida dessa área, zelando ainda para o cumprimento das leis relacionadas ao SUS, principalmente no que tange aos direitos à assistência à saúde de qualidade, à universalidade do acesso, a integralidade e a equidade. $\mathrm{O}$ que ainda representa um desafio para os serviços públicos de saúde em todo o país (BRASIL, 2013; RAMOS et al., 2012).

A atuação desses conselheiros se torna proeminente ao se considerar os desafios encontrados atualmente para a oferta de serviços públicos de saúde de qualidade no país, com destaque para os serviços da Atenção Primária à Saúde (APS). A APS representa é o foco (re) orientador do modelo de atenção à saúde no Brasil. Sua proposta tem a atuação de Equipes de Saúde da Família (ESF) como principal estratégia para implementar seus serviços. Essas equipes multiprofissionais tem seu processo de trabalho baseado na vigilância, a promoção e proteção da saúde, o diagnóstico precoce, o tratamento e a recuperação da saúde dirigida aos indivíduos, à família e à comunidade (BRASIL, 2012a; DUARTE; SOUZA; CLAUDINO, 2011).

Com as ESFs, a APS se expandiu de maneira significativa por todo o país, estando presente em todos os

DOI: https://doi.org/10.25110/arqsaude.v19i3.2015.5551

${ }^{1}$ Enfermeira - Universidade Federal dos Vales do Jequitinhonha e Mucuri. E-mail: karenabarroso@hotmail.com.

${ }^{2}$ Doutorando Faculdade Medicina da UFMG. Professor Assistente do Departamento de Enfermagem da Universidade Federal dos Vales do Jequitinhonha e Mucuri. Diamantina, Brasil. E-mail georgesobrinho@yahoo.com.br 
municípios brasileiros. Esta expansão ampliou o acesso da população aos serviços de saúde, implicando diretamente na melhoria de muitos indicadores de saúde no país. Porém, apesar dessas conquistas, a APS ainda apresenta grandes desafios. Sua cobertura populacional ainda gira em torno de $63,12 \%$ da população brasileira, em que cerca de $80 \%$ desta dependam exclusivamente do SUS. Além disso, muitos serviços são reconhecidos pelas suas dificuldades organizacionais e baixa qualidade, o que implica, muitas vezes, na insatisfação de gestores, profissionais e população que utilizam esses serviços (BRASIL, 2015; SILVA, 2009).

Considerando que em muitas localidades do país estes serviços de saúde significam o único equipamento social existente, acessível e disponível de forma gratuita para a população, ou seja, a única representação e presença do Estado como garantidor dos direitos sociais que a população reconhece, torna-se fundamental que estejam aptos a oferecerem serviços de qualidade e que atendam às devidas necessidades de suas respectivas populações, daí a importância do controle social (BRASIL, 2007).

Contudo, para que o controle social funcione e cumpra com objetivos pelo qual foi criado, há a necessidade de ser ter conselheiros devidamente habilitados e comprometidos em desempenhar suas funções. Esses devem deter o conhecimento das necessidades das comunidades que representam. Além disso, devem compreender a funcionalidade dos serviços de saúde para que possam contribuir para a melhoria da qualidade dese seguimento (RAMOS et al 2013). Desta forma, o presente estudo tem por objetivo analisar a atuação de conselheiros municipais de saúde no controle social em serviços de Atenção Primária à Saúde.

\section{Metodologia}

Trata-se de um estudo transversal com abordagem quantitativa do tipo descritivo realizado junto aos conselheiros municipais de saúde de quatro municípios da microrregião sanitária de Diamantina (Datas, Diamantina, Senador Modestino Gonçalves e Presidente Kubitscheck), todos localizados na região do Vale do Jequitinhonha em Minas Gerais, Brasil. Foram entrevistados 40 conselheiros de saúde, representando $100 \%$ dos conselheiros ativos no conjunto de municípios no período estudado.

Foram incluídos os conselheiros que estavam com seus mandados em vigor e que exerceram o cargo por pelo menos seis meses. Excluíram-se os conselheiros que não foram localizados após três tentativas.

Para a coleta de dados utilizou-se um questionário construído com base no estudo de Avritzer (2009), acrescido de outras questões elaboradas pelo próprio pesquisador. Totalizaram 30 perguntas objetivas relacionadas ao perfil dos conselheiros e sua atuação nos respectivos municípios. Anteriormente à aplicação do questionário, realizou-se pré-teste junto a 15 conselheiros de um município da mesma microrregião para avaliar a adequação de suas questões ao cenário estudado. Esses dados não fizeram parte da pesquisa.

Os dados foram analisados empregando-se estatística descritiva, utilizando o software SPSS, versão 18.0 e Microsoft Excel. As variáveis qualitativas foram descritas por meio de frequência absoluta e relativa e apresentados em tabelas.
A realização da pesquisa obedeceu todos os preceitos éticos exigidos pela Resolução 466/12 do Conselho Nacional de Saúde. A presente pesquisa foi aprovada pelo Comitê de Ética e Pesquisa da Universidade Federal dos Vales do Jequitinhonha e do Mucuri - CEP/UFVJM, Parecer número 033/12.

\section{Resultados}

Os conselheiros que participaram deste estudo eram preponderantemente do gênero feminino (72,5\%), casados $(67,5 \%)$ e com ensino superior completo/ incompleto (52,5\%). Parte significativa dos conselheiros $(35,0 \%)$ eram funcionários públicos concursados do próprio município, conforme apresentado na Tabela 1.

Tabela 1: Perfil de identificação dos conselheiros municipais de saúde de quatro municípios da microrregião sanitária de Diamantina, Minas Gerais, Brasil.

\begin{tabular}{lrr}
\hline Variáveis & $\mathbf{N}$ & $\mathbf{\%}$ \\
\hline Gênero & & \\
Feminino & 29,0 & 72,5 \\
Masculino & 11,0 & 27,5 \\
\hline Estado Civil & & \\
Solteiro & 6,0 & 15,0 \\
Casado & 27,0 & 67,5 \\
Divorciado/separado & 1,0 & 2,5 \\
Viúvo & 2,0 & 5,0 \\
Não respondeu & 4,0 & 10,0 \\
\hline Escolaridade & & \\
Analfabeto & 0 & 0 \\
Sabe ler e escrever, não frequentou escola. & 1,0 & 2,5 \\
Básico & 8,0 & 20,0 \\
Fundamental & 1,0 & 2,5 \\
Médio & 8,0 & 20,0 \\
Superior completo / incompleto & 21,0 & 52,5 \\
Não respondeu & 1,0 & 2,5 \\
\hline Situação ocupacional & & \\
Inativo & 7,0 & 17,5 \\
Desempregado & 2,0 & 5,0 \\
Empregado com carteira & 2,0 & 5,0 \\
Empregado com contrato de trabalho & 6,0 & 15,0 \\
Funcionário público concursado & 14,0 & 35,0 \\
Funcionário público/cargo de confiança & 3,0 & 7,5 \\
Autônomo & 4,0 & 10,0 \\
Free lancer & 2,0 & 5,0 \\
\hline
\end{tabular}

Fonte: Dados da pesquisa.

Em relação às proporções dos segmentos da sociedade representados pelos conselheiros entrevistados observou-se 12,0 (30,0\%) trabalhadores da saúde, 11,0 (27,5\%) de usuários, 5,0 (12,5\%) prestadores de serviços de saúde, $3,0(7,5 \%)$ governo e $9,0(22,5 \%)$ representam outro tipo de segmento.

A principal forma de eleição dos conselheiros para os conselhos de saúde foi via indicação pelas Secretarias Municipais de Saúde 15,0 (37,5\%), seguido de indicação por alguma associação/entidade da qual participa 15,0 (37,5\%). Apenas 3,0 (7,5\%) relataram terem sidos eleitos em Conferências de Saúde, e outros 3,0 (7,5\%) foram eleitos em processo interno da associação/entidade que representam.

No que se refere à participação nas reuniões dos Conselhos de Saúde, a maioria dos conselheiros 35,0 (87,5\%) considerou-se participativa e ter conhecimento prévio das pautas das reuniões. Outros 5,0 (12,5\%) afirmaram que somente, às vezes, ficavam sabendo das pautas. A principal forma de convocação para as reuniões foi por meio de convites 
dos conselhos, $(75,0 \%)$. Outros $2,5 \%$ disseram que as datas das reuniões já eram previamente acertadas. Em relação aos temas das reuniões dos conselhos 21,0 (52,5\%) relataram os discutir previamente com as respectivas entidades que representam. 22,0 (55,0\%) dos conselheiros afirmaram que sempre divulgavam suas ações tomadas durante as reuniões. Para $23(57,5 \%)$ essas informações eram repassadas para a população por meio de conversas com integrantes do segmento/ comunidade que representam de acordo com a tabela 2.

Os resultados mostram também que 31,0 (77,5\%) afirmaram votar de acordo com o conhecimento pessoal dos assuntos. Cerca de 23,0 (57,5\%) já fizeram alguma proposta ao conselho, porém a maioria $(52,5 \%)$, nunca conseguiu que nenhuma proposta implementada. Os conselheiros ainda consideraram que a baixa presença nas plenárias 16,0 (40,0\%) é o principal fator limitante para que as ações dentro dos conselhos aconteçam, ainda assim, 20 (50,0\%) desses consideram que o funcionamento de seus respectivos conselhos é considerado bom.

Tabela 2: Perfil de atuação dos conselheiros municipais de saúde de quatro municípios da microrregião sanitária de Diamantina, Minas Gerais, Brasil.

\begin{tabular}{|c|c|c|}
\hline Variáveis & $\mathrm{N}$ & $\%$ \\
\hline \multicolumn{3}{|l|}{ Discute temas antes da reunião } \\
\hline Sim. Nas reuniões da entidade que participo & 21,0 & 52,5 \\
\hline Sim. Nas reuniões somente quando é interesse do grupo & 3,0 & 7,5 \\
\hline Sim. Informalmente com os membros da entidade & 8,0 & 20,0 \\
\hline Não discuto previamente & 4,0 & 10,0 \\
\hline Raramente discuto & 2,0 & 5,0 \\
\hline Não respondeu & 2,0 & 5,0 \\
\hline \multicolumn{3}{|l|}{ Divulga ações para a sociedade } \\
\hline Sempre & 22,0 & 55,0 \\
\hline Às vezes & 12,0 & 30,0 \\
\hline Raramente & 2,0 & 5,0 \\
\hline Nunca & 3,0 & 7,5 \\
\hline Não respondeu & 1,0 & 2,5 \\
\hline \multicolumn{3}{|l|}{ Forma de divulgação das ações e decisões } \\
\hline Reuniões periódicas com comunidade ou segmento próprias para divulgação & 4,0 & 10,0 \\
\hline Informes impressos (artigos em jornal próprio, boletins, murais) & 0 & 0 \\
\hline Meios de comunicação mais amplos (jornais, revistas, rádios) & 3,0 & 7,5 \\
\hline Conversa com integrantes do segmento/comunidade que representa & 23,0 & 57,5 \\
\hline Não tem o hábito de divulgar as informações para o segmento/comunidade & 8,0 & 20,0 \\
\hline Não respondeu & 2,0 & 5,0 \\
\hline \multicolumn{3}{|l|}{ Vota de acordo } \\
\hline Sua opinião e conhecimento pess oal sobre o as sunto em questão & 31,0 & 77,5 \\
\hline Orientação da entidade ou segmento que representa & 3,0 & 7,5 \\
\hline Orientação da câmara técnica ou do grupo/comissão de trabalho & 3,0 & 7,5 \\
\hline Outro & 2,0 & 5,0 \\
\hline Não respondeu & 1,0 & 2,5 \\
\hline \multicolumn{3}{|l|}{ Dificuldade para ação do conselho } \\
\hline Baixa presença de conselheiros nas plenárias & 16,0 & 40,0 \\
\hline Baixa presença de repres entantes do governo nas plenárias & 0 & 0 \\
\hline Centralização do poder na secretaria/ prefeitura & 1,0 & 2,5 \\
\hline Pouca rotatividade entre os membros dos conselhos & 2,0 & 5,0 \\
\hline Muita rotatividade entre os membros dos conselhos & 1,0 & 2,5 \\
\hline $\begin{array}{l}\text { Precariedades das instalações físicas dos conselhos e/ou falta de organização interna } \\
\text { A mesa diretora é muito centralizadora }\end{array}$ & 3,0 & 7,5 \\
\hline Outro & 1,0 & 2,5 \\
\hline \multirow[t]{2}{*}{ Não respondeu } & 6,0 & 15,0 \\
\hline & 10,0 & 25,0 \\
\hline
\end{tabular}

Fonte: Dados da pesquisa

Quanto ao nível de informação dos entrevistados a respeito dos serviços de saúde existentes nos respectivos municípios, 25,0 (62,5\%) responderam que conhecem todos os serviços prestados, 9,0 (22,5\%) conhecem apenas alguns e 5,0 (12,5\%) não sabem quais são os serviços existentes no próprio município.

No que diz respeito às capacitações para desem- penhar as atividades de conselheiro observou-se que 57,5\% nunca participaram de nenhum tipo de capacitação, o que reflete diretamente no conhecimento em relação as leis que regem o controle social, em que 50,0\% afirmaram conhecer apenas em partes estas leis, enquanto que outros $15,0 \%$ relataram não ter nenhum conhecimento sobre elas.

Nesta pesquisa foram feitas também questões a res- 
peito da relação dos conselheiros e a Atenção Primária à Saúde. Nesta viu-se que 75,0\% dos conselheiros afirmaram saber do significado e dos objetivos da Atenção Primária nos serviços de saúde. Em relação à ESF, 87,5\% são usuários destes serviços, $80,0 \%$ conhecem ou sabem identificar os profissionais da equipe em que são cadastrados e $75,0 \%$ conhecem bem quais serviços são prestados por este serviço.

No que tange ao conhecimento dos problemas e limitações da unidade de saúde, $22(55,0 \%)$ afirmaram os conhecer e $60,0 \%$ atua levando estes problemas para serem debatidos junto aos conselhos de saúde, conforme dados apresentados na Tabela 3:

Tabela 3: Relação dos conselheiros municipais de saúde e a atenção primária a saúde de quatro municípios da microrregião sanitária de Diamantina, Minas Gerais, Brasil.

\begin{tabular}{|c|c|c|}
\hline Variáveis & $\mathrm{N}$ & $\%$ \\
\hline \multicolumn{3}{|l|}{ Conhece os problemas do seu Pos to de Saúde ou ESF } \\
\hline Sim & 22,0 & 55,0 \\
\hline Não & 8,0 & 20,0 \\
\hline Alguns & 7,0 & 17,5 \\
\hline Não respondeu & 3,0 & 7,5 \\
\hline \multicolumn{3}{|c|}{$\begin{array}{l}\text { O que você tem feito pra mudar ou corrigir os problemas de saúde do seu Pos to de saúde } \\
\text { ou ESF }\end{array}$} \\
\hline Levo os problemas de saúde para serem pautas nas reuniões dos conselhos & 24,0 & 60,0 \\
\hline Não fico sabendo dos problemas de saúde do Posto de Saúde ou ESF & 5,0 & 12,5 \\
\hline Não me envolvo com os problemas de saúde do Posto de Saúde ou ESF & 2,0 & 5,0 \\
\hline Outro & 1,0 & 2,5 \\
\hline Não respondeu & 8,0 & 20,0 \\
\hline
\end{tabular}

Fonte: Dados da pesquisa

Os dados mostram que os conselheiros não têm uma participação na discussão dos problemas ou dos aspectos organizacionais nas unidades de saúde. Os dados mostram que $32,5 \%$ responderam que não participam das reuniões feitas na unidade de saúde. Outros 30,0\% participam pouco e apenas $25,0 \%$ disseram sempre participarem de reuniões na ESF. Contudo, apesar desta limitada atuação junto às equipes, $82,5 \%$ dos conselheiros afirmaram que os serviços de saúde prestados pelas unidades de saúde eram considerados bom ou muito bom e 7,5\% os avaliaram como regular.

\section{Discussão}

\section{O perfil dos Conselheiros de Saúde}

A análise dos dados revela que o perfil dos conselheiros de saúde se destaca por ser composto, em sua maioria, de pessoas que possuem curso superior completo. O que por um lado, é considerado positivo por Bezerra e Araújo (2009) pela possibilidade de apresentarem melhor conhecimento e capacidade de terem um posicionamento mais crítico em relação às políticas públicas. Porém, Ramos et al (2012) relatam que este contexto não significa uma correlação de forças dentro do Conselho. Há também, a necessidade de se considerar a experiência e o saber local, garantindo que mesmo aqueles sem educação formal sejam ouvidos, pois certamente podem ser conhecedores de questões importantes de sua comunidade.

Outros achados sobre o perfil dos conselheiros dizem respeito à ocupação dos conselheiros, em que 34,0\% são funcionários públicos da secretaria de saúde do respectivo município. Achado semelhante é encontrado em um estudo realizado em seis municípios do estado de São Paulo de Zambon e Ogata (2011), em que, mesmo na ocorrência de processos eleitorais, a maioria dos representantes dos conselhos acabara sendo composta por profissionais das próprias Secretarias de Saúde, incluindo o próprio o secretário de saú- de muitas vezes eleito como seu presidente.

Estudos apontam que muito frequentemente a composição dos Conselhos de Saúde acontece apenas pela sua exigência legal, em que, pela ausência da sociedade, seja por não conhecer ou por ser excluída dos processos de participação social, permitem que estes Conselhos sejam compostos por representantes que não foram eleitos pelo processo democrático preconizado em lei (BRASIL, 2012b). Isso contribui para a baixa rotatividade dos conselheiros e compromete o seu papel fiscalizador e deliberativo. Além disso, fazem dos atos dos conselheiros meros rituais de legitimação de políticas e decisões supostamente democráticas, afetando cada vez mais o estímulo ao fortalecimento da participação da população na formulação e implementação de políticas públicas, bem como a transparência dos serviços de saúde prestados a sociedade. Esses achados demonstram a influência do governo local na composição dos Conselhos, o que fica mais evidente quando se observa que a maioria dos conselheiros teve indicação pela respectiva Secretaria de Saúde e a minoria eleita conforme preconizado em lei(OLIVEIRA; ALMEIDA, 2002).

A alteração da paridade da representação social dos Conselhos de Saúde pode comprometer as decisões ali tomadas, isso por possibilitar a influência da gestão local, seja pela possibilidade de distorção da realidade percebida diante dos interesses ali representados. Isso pode contribuir diretamente para a prática de decisões clientelistas e a ausência da cultura de participação e controle social, uma vez que se possa exercer o controle social pela população, desenvolvido pelos Conselhos Municipais de Saúde, para que se possa buscar a consolidação da reorientação do modelo de atenção com enfoque na Atenção Primária consoante às necessidades de saúde, é fundamental se ter uma representação forte dos seus usuários (PEREIRA, 2008; MARTINS et al 2008) 


\section{A Atuação dos Conselheiros de Saúde}

Quanto à atuação dos conselheiros observou-se que os conselheiros alegaram possuir bons mecanismos de convocação para as reuniões, como o conhecimento prévio das datas e das pautas, principalmente mediante cartas-convite, $\mathrm{o}$ que retrata a capacidade organizativa dos Conselhos e que é essencial para a sua boa funcionalidade, tornando mais transparente o seu trabalho e o vínculo com a população (GRISOTTI; PATRICIO; SILVA, 2010; VIEIRA; CALVO, 2011).

Os conselheiros ainda reportam sobre a discussão prévia e posterior dos temas das reuniões com seus seguimentos, o que se pode considerar positivo, já que denota que a atuação do Conselho ressoa nas instituições, categorias e comunidades, dando a elas conhecimento das deliberações do colegiado, a possibilidade de expor suas necessidades e fortalecendo o vínculo com a população (DUARTE, MACHADO, 2012).

Entretanto, encontram-se alguns pontos que evidenciam limitações na atuação dos conselheiros, como a baixa presença destes nas plenárias, conforme apontado por $40,0 \%$ deles, o que contraria a afirmação de $87,5 \%$ se considerarem bem participativos no desempenho da atividade de conselheiros. A ausência dos colegas nas reuniões é apontada por esses professores como principal fator limitante para a efetiva funcionalidade do Conselho.

Dado semelhante foi encontrado no estudo de Batista et al. (2010), que aponta a baixa participação de conselheiros em reuniões como um problema dificultador no processo de consolidação do Conselho como espaço para o exercício da democracia e concretização dos princípios do SUS. Contudo, outros estudos (LANDERDHAL et al., 2010; SALIBA et al., 2009) apontam algumas explicações para tal. Talvez, esta possa ser justificada pela insatisfação dos conselheiros, devido ao fato de que na maioria dos assuntos deliberados são oriundos da Secretaria de Saúde e não de uma demanda levantada pelo próprio Conselho de Saúde ou pela população, interferindo assim no poder de decisão, impossibilitando estes conselheiros de reconhecer seu papel no controle social e desvalorizando a representação social. Entretanto, Conta et al (2011) apontam que esta ausência também pode se dar por certo descompromisso e desorganização destes com relação as suas atribuições e deveres como representantes legais da sociedade.

Apesar de avaliarem bem o seu próprio desempenho, poucos conselheiros propuseram ou têm propostas próprias aprovadas nas reuniões, o que denota uma fragilidade ou limitação do controle social ali exercido. Propor significa conhecimento da realidade em que está inserida, dos problemas vivenciados e até mesmo conhecimento do seu papel e compromisso enquanto conselheiro (SALIBA et al., 2009)

Outro ponto que chama a atenção é o fato de $57,5 \%$ dos conselheiros nunca terem participado de nenhuma capacitação para desempenhara função. Isso reflete como evidenciado, no desconhecimento das leis que regem o controle social e o próprio entendimento da funcionalidade dos serviços de saúde. Partindo do pressuposto que só se pode controlar aquilo que se conhece, Silva et al (2012) afirmam que, para que o conselheiro de saúde possa exercer efetivamente o seu papel é necessária uma educação permanente para que ele conheça a legislação que o ampara e que garante democrati- camente sua participação. Outros estudos reportam que essa falta de qualificação dos conselheiros é comum em outras localidades e destacam que essa é uma necessidade sentida por cerca de $85 \%$ dos conselheiros entrevistados (BEZERRA, ARAUJO, 2009; COTTA, CAZAL, RODRIGUES, 2009).

\section{Relação com a Atenção Primaria à Saúde}

Na relação dos conselheiros com a Atenção Primária à Saúde procurou inicialmente saber sobre o entendimento deles sobre este serviço e seus objetivos. Assim, 75,0\% dos conselheiros afirmaram terem bom conhecimento sobre o conceito e os objetivos da APS, o que é importante para o desempenho do controle social junto a estes serviços. O entendimento da funcionalidade dos serviços pode o credenciar para a melhor identificação de limitações e necessidades como na proposição de soluções dessas com os profissionais, a gestão e a comunidade. A Política Nacional da Atenção Básica ainda reforça a necessidade das equipes de saúde, sobretudo no âmbito das Equipes de Saúde da Família, de promoverem a educação contínua da população sobre sua funcionalidade, o que é visto como importante mecanismo para potencializar o exercício do controle social na busca da qualidade destes serviços (VIEIRA; CALVO, 2011; BRASIL, 2013).

Os conselheiros relatam que possuem um bom conhecimento sobre os profissionais e a funcionalidade dos serviços de saúde em que são usuários. Isso mostra a aproximação destes com os serviços, o que é importante no desempenho do seu papel (CREVELIM, PEDUZZI, 2005). No entanto, os dados mostram que $62,5 \%$ dos conselheiros não participam ou participam pouco de reuniões nos serviços em que são usuários. Esse dado que pode contradizer o fato de relatarem conhecer bem os serviços de saúde e de haver discussão prévia sobre as decisões dos Conselhos. Tal observação reporta mais uma vez ao déficit na qualificação dos conselheiros, visto que dentre suas atribuições, conforme preconizado em lei, é acompanhar o desenvolvimento das ações e serviços de saúde, conhecer a realidade de sua comunidade para que seja alcançado o direito a saúde, integral e universal do cidadão (BRASIL, 2010).

\section{Conclusão}

A análise aqui empreendida permitiu conhecer a composição e o funcionamento dos quatros Conselhos Municipal de Saúde da microrregião sanitária de Diamantina, possibilitando concluir que embora os conselhos demonstrem uma boa capacidade de organização, sua capacidade de realizar o controle social da forma como é preconizada encontra-se comprometida pela pouca participação da população.

Embora os conselheiros afirmem conhecer a APS e os serviços de saúde do seu município, pode-se perceber que não participam das ações dentro destes próprios serviços. Dessa maneira, a falta de participação dos conselheiros pode indicar a existência de barreiras que ainda precisam ser superadas para que o controle social se torne efetivo no seu papel de trabalhar em prol da melhoria continua dos serviços de APS e no atendimento das necessidades dos usuários.

As limitações deste estudo residem no fato de facalizarmos apenas o ponto de vista dos conselheiros. Tendo 
em vista que para o entendimento de como se dá o controle social nestes serviços caberia também os pontos de vista de gestores, comunidade e trabalhadores da saúde. Assim, este estudo aponta para a necessidade de se aprofundar no estudo do tema aqui presentado. Sobretudo, de se buscar conhecer melhor as dinâmicas que envolvem o exercício do controle social nos municípios, tanto no âmbito dos conselhos como nos próprios serviços de saúde e até na comunidade.

\section{Referências}

AVRITZER, L et al. Democracia, Desigualdade e Políticas Públicas no Brasil. Projeto Democracia participativa. v.2. Belo Horizonte. 2009.

BATISTA, A.A. et al. A Contribuição da Pesquisa Avaliação para o Processo de Implementação do Controle Social no SUS. Saúde Soc.v.19, n. 4, p. 784-793, 2010.

BEZERRA, C.K.F.; ARAÚJO, M.A.D. Conselho Municipal de Saúde de Pedras do Fogo - PB: um estudo sobre participação. Rev. Adm. Saúde. v.11, n. 42, 30-42, 2009.

BRASIL. Ministério da Saúde. Conselho Nacional de Secretários de Saúde. Atenção Primária e Promoção da Saúde. Brasília: CONASS, 2007.

Tribunal de Contas da União. Orientações para conselheiros de saúde. Brasília: TCU, 4ª Secretaria de Controle Externo, 2010.

Ministério da Saúde. Resolução CNS n-0 453, de 10 de maio de 2012. Aprovar as seguintes diretrizes para instituição, reformulação, reestruturação e funcionamento dos Conselhos de Saúde. Diário Oficial da União n 109. Brasília, DF 10 mai.; Seção 1. p. 138, 2012a.

Ministério da Saúde. Secretaria de Atenção à Saúde. Política Nacional de Atenção Básica. Brasília (DF): Ministério da Saúde, 2012b.

Ministério da Saúde. Conselho Nacional de Saúde. Para entender o controle social na saúde. Brasília: Ministério da Saúde, 2013.

Ministério da Saúde. Departamento de Atenção Básica [ on-line]. [Citado em 2015 set 15]. Disponível em: URL: http://dab.saude.gov.br/dab/historico_cobertura_sf/ historico_cobertura_sf_relatorio.php.

Lei no 8.142, de 28 de Dezembro de 1990.

Dispõe sobre a participação da comunidade na gestão do Sistema Único de Saúde (SUS) e sobre as transferências intergovernamentais de recursos financeiros na área da saúde e dá outras providências [on-line]. Brasília, DF; 1990. [Citado em 2015 jan. 22]. Disponível em: URL:http:// www.planalto.gov.br/ccivil_03/leis/18142.htm.

CORREIA, M.V.C. O conselho Nacional de Saúde e os Rumos da Política de Saúde Brasileira: mecanismo de controle social frente às condicionalidades dos organismos financeiros internacionais. 2005. 324f. Tese (Doutorado serviço social) - Universidade Federal de Pernambuco, Pernambuco, 2005.

COTTA R.M.M. et al. O controle social em cena: refletindo sobre a participação popular no contexto dos Conselhos de saúde. Physis. v. 21, n. 3, p. 1121-1137, 2011.

COTTA, R.M.M.; CAZAL, M.M.; RODRIGUES, J.F.C. Participação, Controle Social e Exercício da Cidadania: a (des) informação como obstáculo à atuação dos conselheiros de saúde. Physis. v.19, n. 2, p. 419-438, 2009.

CREVELIM, M.A.; PEDUZZI, M.A. Participação da comunidade na equipe de saúde da família: Como estabelecer um projeto comum entre trabalhadores e usuários? Cien. Saúde Colet.; v.10, n. 2, 2005

CRISTO, S. C. A. Controle social em saúde: o caso do Pará. Serv. Soc. Soc., São Paulo, n. 109, p. 93-111, 2012.

DUARTE, E.B.; MACHADO, M.F.A.S. O Exercício do Controle Social no Âmbito do Conselho Municipal de Saúde de Canindé, CE. Saúde Soc. v. 21, n.1, p. 126-137, 2012.

DUARTE, S.J.H.; SOUZA, N.F.; CLAUDINO, T.X. Relato de experiência acerca do trabalho do Agente Comunitário de Saúde: reflexões sobre sua prática. Gestão e Saúde, v.2, n.1, p.173-183, 2011.

GRISOTTI, M.; PATRÍCIO, Z.M.; SILVA, A. A participação de usuários, trabalhadores e conselheiros de saúde: um estudo qualitativo. Cien. Saúde Colet, v.15, n.3, p.831-840, 2010.

LANDERDHAL, M.C. et al. Resoluções do Conselho de Saúde: instrumento de controle social ou documento burocrático? Cien. Saúde Colet. v.15, n. 5, p. 2431-2436, 2010 .

MARTINS, P.C. et al. Conselhos de saúde e a participação social no Brasil: matizes da utopia. Physis. v,18, n. 1, p.105-121, 2008

OLIVEIRA, M.L.; ALMEIDA, E.S. Controle Social e Gestão Participativa em Saúde Pública em Unidades de Saúde do Município de Campo Grande, MS, 1994-2002. Saúde Soc. v.18, n. 1, p. 141-153., 2002.

PEREIRA, M.F. Mundos Sociais: Saberes e Práticas. Experiência de Participação Pública nos Conselhos Municipais de Saúde no Brasil. In: VI CONGRESSO PORTUGUÊS DE SOCIOLOGIA, 2008, Lisboa. Anais... Universidade Nova de Lisboa. Faculdade de Ciências Sociais e Humanas. P. 788, 2008.

RAMOS, F.R. et al. Conselhos Setoriais: perfil dos conselheiros e sua influência na tomada de decisão. Saúde Soc. São Paulo, v.21, n.3, p.61-70, 2012. 
SALIBA, N.A. et al. Conselhos de saúde: conhecimento sobre as ações de saúde. Rap. v. 43, n.6, p. 1369-1378, 2009.

SHIMIZU, H. E. et al. Representações sociais dos conselheiros municipais acerca do controle social em saúde no SUS. Cienc. Saúde Colet, v.18, n.8, p. 2275- 2284, 2013.

SILVA, G.S. O processo de trabalho do coordenador municipal da estratégia de saúde da família. 2009. 96f. Dissertação (Mestrado em Enfermagem). Escola de Enfermagem, Universidade Federal de Minas Gerais, Belo Horizonte, 2009.

SILVA L.M.S. Et al. Análise da Organização e funcionamento dos Conselhos de Saúde e a Gestão Participativa em Fortaleza, CE. Saúde Soc. v. 21, n. 1, p. 117-125, 2012.

VIEIRA, M.; CALVO, M.C.M. Avaliação das condições de atuação de Conselhos Municipais de Saúde no Estado de Santa Catarina, Brasil. Cad. Saúde Pública. v. 27, n.12, p.2315-2326, 2011.

ZAMBON, V.D.; OGATA, M.N. Configurações dos Conselhos Municipais de Saúde de uma região no Estado de São Paulo. Rev Esc Enferm USP, v.45, n.4, p.890-897, 2011.

Recebido: 03/02/2015 Aceito: 03/11/2015 